\title{
Non-Monte Carlo Methods for Investigating the Application of Coded Aperture Breast Tumour Imaging
}

Alnafea MA*, Mahboub D and Wells K

Department of Radiological Sciences, College of Applied Medical Sciences, Riyadh, Saudi Arabia

\begin{abstract}
This is a continuous investigation of a possible application of Coded Aperture (CA) for breast tumor imaging. This image formation system allows a high photon transmission, image magnification and to a lesser extent provide (limited-angle) tomographic capability. This paper describes two non-Monte Carlo methods, the first one based on a simple attractive approach called Binary Mask Shift (BMS) representing the action of a distributed source in the projective CA imaging geometry. It allows to investigate all the possible built in artefacts without the effect of solid angle. Thus, it provides understanding of the application of CA patterns in breast tumor imaging through the evaluation of their potential and their performance under a variety of imaging conditions. The second method based on Pseudo-Ray Tracing (PRT) that obtained by purely calculating the angle of incidence of each point in the object that successfully strikes an open aperture element and then hits the detector element. These methods particularly used for CA imaging investigations. Interestingly, these methods yield similar results of a similar CA pattern but takes less computing power, than using a full Monte Carlo Simulation (MCS) approach. The main results demonstrate that Modified Uniformly Redundant Arrays (MURAs) patterns of squares arrays are very promising as it produces excellent decoded images. This is attractive particularly when used for breast tumor imaging i.e. ideal for imaging small isolated sources and thus provides a good match to the imaging of Scintimammography (SM).
\end{abstract}

Keywords: Binary mask shift; Pseudo-ray tracing; Coded aperture; Breast tumor imaging

\section{Introduction}

Breast cancer is a disease that cause cells in the breast to change and grow abnormally out of control. It primarily effects women but about $1 \%$ of all cases effect men as well. It is rare in women below the age of twenty years and less common below the age of thirty years, but it is more aggressive and thus has a lower survival rate. The incidence rate however, still rises with age every year as and thus, considered the second most common form of cancer among women worldwide [1]. As well as it is the second leading cause of death in women next to lung cancer in the United States (US). Statistics $[1,2]$ show that one out of eight women $(12.2 \%)$ in the US will develop breast cancer in their lifetime and unfortunately one third of those women die from it. Additionally, the high prevalence of breast cancer in Western populations has led to a high degree of public awareness and involvement. As a result of this intense interest, prompted global governments to put constant efforts and quality driven to a greater extent than any other disease to increase patient's recovery level against this group of disease.

Most medical professionals are familiar with the role of imaging in cancer detection. Breast cancer typically detected either before symptoms appears through clinical screening or examination, or after symptoms appears i.e. woman notices a lump in their breast tissues. Mammography uses X-ray to try to find breast cancer through screening before a lump can be felt. The goal is to detect and consequently treat cancer early, when a cure is more likely. Most masses seen on X-ray mammogram turn out to be benign breast lumps i.e. are not life threatening. However, for a definitive diagnosis and to determine the extent of spread and characterize the type of the disease tissue microscopic analysis need. This often obtained via a needle or surgical biopsy. The type of biopsy is based on individual patient clinical factors, availability of particular biopsy devices, and resources.

Breast imaging facilities and professionals are subject to specific accreditation requirements. The demanding breast health environment creates opportunities and challenges for developers of breast devices.
The role of imaging in breast health environment divided into clinical and research roles. As well as, detection require imaging tools for research and clinical practice. The majority of reported literature suggested that the screening for breast cancer likely reduces breast cancer mortality, but the magnitude of the effect is uncertain. Screening will also result in some women getting a cancer diagnosis even though their cancer would not have led to death. Furthermore, women will experience pain, stress and other psychological distress for many months because of false positive findings. Complementary imaging modalities such as Magnetic Resonance Imaging (MRI) has been recommended as a first-line tool in women considered to be at high risk of developing breast cancer. Unfortunately, large-scale clinical trials have shown that the accuracy of MRI is probably inadequate reliably detect breast of all lesions for this application. The same applied to the breast ultrasonography imaging modality.

\section{The role of scintimammography}

Scintimammography (SM) is a promising functional radionuclide imaging technique that is generally undertaken using high resolution parallel-hole collimators with Anger cameras. The performance of the general purpose clinical gamma camera in lesion detection using ${ }^{99 \mathrm{~m}} \mathrm{Tc}$ Sestamibi, is dependent on tumour size. Imaging patients with lesions of sizes $>1 \mathrm{~cm}$ in diameter are more likely to be detected. This mean that the sensitivity is higher for lesion $>1 \mathrm{~cm}$ in diameter than smaller lesion.

*Corresponding author: M. A. Alnafea, Department of Radiological Sciences, College of Applied Medical Sciences, Riyadh 11433, P.O. Box 10219, Saudi Arabia Tel: 966549009 006; E-mail: alnafea@ksu.edu.sa

Received September 21, 2017; Accepted December 27, 2017; Published December 29, 2017

Citation: Alnafea MA, Mahboub D, Wells K (2017) Non-Monte Carlo Methods for Investigating the Application of Coded Aperture Breast Tumour Imaging. J Cancer Sci Ther 9: 748-754. doi:10.4172/1948-5956.1000503

Copyright: (c) 2017 Alnafea MA, et al. This is an open-access article distributed under the terms of the Creative Commons Attribution License, which permits unrestricted use, distribution, and reproduction in any medium, provided the original author and source are credited. 
Similarly, the sensitivity of lesion detection is higher for palpable lesion than non-palpable lesion. However, the technique becomes less reliable in lesions $<1 \mathrm{~cm}$ in diameter [3] may be due to the small incident flux from such lesions. In this case photon noise may dominate the image and consequently reduce image resolution. The significant noise from the background activity further complicate the detection. This technique suffers from some clinical limitations as it is less reliable at detecting small (less than $1 \mathrm{~cm}$ in diameter) lesions. These limitations are due to resolution-efficiency trade-off that is inherent in the use of collimation. In Saudi, a large proportion of women screened by digital X-ray mammography have dense breasts particularly in women below the age of 45 years. This group of patients are advised to have a test that independent of breast density. SM imaging is the modality recommended for this group of patients, as it is not affected by the density of the breast as it has a higher sensitivity over mammography. This modality plays an important role, as a complementary imaging method to X-ray mammography, for many different clinical applications. In addition, the last two decades have seen tremendous efforts by scientists to develop advanced radionuclide imaging instrumentation dedicated for breast imaging to aid the conventional method. Surgery, radiation therapy, hormonal replacement therapy, excisional biopsies and chemotherapy causes architectural distortion to the breast tissues. As a result, mammographic evaluation in these cases is very difficult. The best alternative is SM as its diagnostic accuracy is not affected by breast tissue distortions. It is also useful in determining the presence of recurrent disease in these circumstances. In North America SM may be regarded as a third line (in some clinical indications may considered as second line) diagnostic aid to assist in the evaluation of breast lesions [4]. SM may also consider to be useful for evaluating non-palpable breast lesions and thus aid X-ray mammography. Using the two imaging modalities has been demonstrated [5] to improve the low positive predictive value (PPV) which may help in detecting non-palpable breast lesions. In addition, SM may also be valuable for the assessment of multifocal breast cancer [6]. Moreover, two clinical studies [7] reported that SM may also be useful for monitoring and evaluating the tumour response to chemotherapy and for investigation of patients with microcalcifications. And also, valuable for detecting axillary lymph nodes metastasis [8]. Taillefer [3] has reviewed the published papers between 1994 and 1998 to investigate the clinical capability and the performance of SM using standard gamma camera. His paper is based on 20 studies with a total of 2009 patient and showed discrepancies in the reported data from these studies. These variations in the reported values was believed to be due to several factors such as different patient population and different type, size, location and tumour uptake. In addition, a variety of other factors related to the camera performance, resolution, settings, acquisition, imaging time and the type and diameter of the collimator used. A summary of the results is given in Table 1. He confirmed that SM may be used effectively as a complementary method to X-ray mammography and showed that no lesion less than $5 \mathrm{~mm}$ has been detected by the standard full-size clinical gamma camera detectors. He also demonstrated that

\begin{tabular}{|c|c|}
\hline Test Parameters & Summary of the results \\
\hline Total average sensitivity & $85 \%(1,029 / 1,218$ lesions $)$ \\
\hline Total average specificity & $89 \%(963 / 1,086$ lesions $)$ \\
\hline Total average accuracy & $86 \%(1,992 / 2,304$ lesions $)$ \\
\hline Total average PPV & $89 \%(1,029 / 1,152$ lesions $)$ \\
\hline Total average NPV & $84 \%(963 / 1,147$ lesions \\
\hline Total average sensitivity & $85 \%(1,029 / 1,218$ lesions $)$ \\
\hline
\end{tabular}

Table 1: A summary of comprehensive review [3] from 20 studies and a total of 2009 patient to evaluate the accuracy of SM. the reported sensitivity varies between $80 \%-90 \%$ and it is higher in the group of patients with palpable lesions than those of non-palpable lesions. The reported average specificity of ${ }^{99 \mathrm{~m}} \mathrm{Tc}-\mathrm{Sestamibi} \mathrm{SM}$ for malignant breast lesions is $89 \%$.

Another study by Itti et al. [9], reported that the overall sensitivity and specificity average $83.3 \%$ and $81.3 \%$, respectively. These results based on the detection of primary breast cancer taking histopathology as the gold standard. In a comparative study published in early 2006 , the Federal Agency for Healthcare Research and Quality (FAHRQ) [10] evaluated the performance of non-invasive diagnostic tests in women with breast abnormalities. These abnormalities were previously identified by either mammography or breast physical examination. This report compares the effectiveness and the diagnostic accuracy of ultrasound, MRI, PET and SM and concluded that none of these non-invasive diagnostics met the suggested standard of having a less than $2 \%$ risk of having cancer particularly among women with negative diagnostic results. However, this is the only negative finding compared to all other studies, nevertheless, provides motivation for work in this study aimed at improving SM imaging performance using CA techniques. In order to improve the sensitivity of SM, many studies [1117] have been performed using dedicated detectors constructed for use in breast tumour imaging applications. Few studies have demonstrated slight improvements in sensitivity with respect to stage I cancers (of size $\leq \mathrm{cm}$ ) when imaging with such high-resolution cameras $[15,17]$. Despite these slight advantages there is still difficulty imaging areas near the thoracic wall or axillary regions because of the small FoV. These cameras are also not reliable to be used for screening purposes because of their limitations in visualizing small calcifications and soft tissue masses $[4,16]$. In addition, a slight reduction in the specificity is shown as the sensitivity increases. The main important limitations in using such dedicated breast cameras are their high cost as well as their role in breast cancer diagnosis are not clearly established. Thus, further studies are necessary to evaluate their complementary role in the management of breast cancer as well as the indications in clinical practice. In summary, most cited papers and review concluded that ${ }^{99 m}$ Tc-sestamibi SM imaging, using full-size clinical gamma camera, is valuable technique and can be effectively used to complement $\mathrm{X}$-ray mammography. These studies suggest that SM is not indicated for breast cancer screening to confirm the presence or absence of malignancy. This technique is not sufficiently accurate to be used as an alternative method to breast biopsies. To be able to do so the diagnostic imaging test must possess a high accuracy so that it can clearly classify and assess the anatomical and physiological features of the image lesion. Thus, ideal imaging test must have both high spatial resolution and high contrast sensitivity with accuracy $>98 \%$ [16]. However, many studies showed that SM might be useful as a complementary imaging technique to improve the sensitivity and specificity of conventional imaging modalities. The main technical problem of the current gammaray detection systems is believed to be due to the use of mechanical collimator. This is because the performance of the standard $\gamma$-camera is heavily dependent on the collimator and its geometry. Since the LEHR collimator is always employed it tend to affect the spatial resolution and the sensitivity of the -camera and the resulting image will have low photon statistics. As a solution to these problems this study proposed using CA, alternative to LEHR collimator, coupled to standard gamma camera for possible breast tumour imaging application. Given that breast care professionals rely heavily on imaging and thus, after careful consideration of the current status of breast imaging tools, we see that there is room for a modality for quantitative functional assessment on a global as well as on a lesion-by-lesion basis, capable of visualizing all stages of tumors. The modality should have a spatial resolution 
on the order of a breast duct's size (i.e., one millimeter), with signal collection efficiency that can support high enough contrast-to-noise ratio to visualize objects of this size. With absolute quantitation and a theoretical intrinsic resolution of one-millimeter, single photon emission computed tomography ("SPECT") would seem like a reasonable choice particularly after replacing the collimator by a CA (an open and close aperture pattern). As an alternative approach, this study hopes to add to this knowledge by proposing using a simple (CA) mask, instead of a collimator coupled to a standard clinical gamma camera for investigating the possible application of CAs for breast tumor imaging. This is an attractive approach as it is simple, inexpensive method since it obtained using full-size clinical gamma camera after removing the collimator. The CAs has an open area significantly greater than the conventional collimator. Thus, provides, potentially, very high sensitivity compared with the parallel-hole collimator. This is particularly attractive at General Hospital level, where the cost of running an additional dedicated imaging system may be prohibitive. In addition, CA imaging as originally developed for astronomical applications, is well suited to detecting faint pseudo-point like objects in a non-zero background; thus, it appears to be well matched to the imaging objectives in SM.

\section{History of coded aperture imaging}

The concept of CA imaging, types and reconstruction methods used to decode and recover the encoded image. The early CAs imaging techniques such as the Fresnel Zone Plate (FZP) [18] and the random array [19] have been avoided in this study as by that these patterns suffer to some degree from distortion artefacts [20,21]. However, the development of CA patterns based on" Cyclic Difference Sets" such as Uniformly Redundant Arrays (URAs) [22-24], Modified Uniformly Redundant Arrays (MURAs) [25] and arrays based on MURAs such as No-Two-Hole-Touching (NTHT) [26] have been demonstrated to be the most promising of all the CA patterns. These optimum arrays, as originally developed and extended by Fenimore and Cannon [23,24,27] have become widely used in the detection of X-ray and gamma ray sources in astronomy for imaging stars and more recently in nuclear medicine for small animal imaging $[28,29]$. These arrays combine the high transmission (have up to $50 \%$ open area for the MURA patterns) characteristic with flat (zero) side-lobes in their response function (Figure 1) The high transmission provides a potential capability to image low-contrast sources and may dramatically enhances the detection efficiency compared to collimators system. Certainly, these advantages and properties have motivated the author to select this family of CA patterns as appropriate for use in SM.

The idea of replacing the conventional collimator by the use of CAs was first employed by Barrett [18]. It basically, involves the placement of CA between the source and the detecting device. Sources at different depths and positions cast a shadows (pattern) of the aperture onto the imaging detector (Figure 2a). Thus, the resultant (projected) image called a composite or multiplexed image (Figure $2 \mathrm{~b}$ ) represents the sum of all projections at different $x$, y positions and depths within the object. To locate the proper size and position of the desired objects, the projected image must be decoded by determining the correlation of the mask pattern in size and position. However, the advantage offered by the CA lies in its increased photon collection efficiency due to its large numbers of open areas i.e. several thousand times that of a single pinhole (Figure 3) [22].

CA imaging system for instance, when imaging a point source (with low statistical noise), in the far-field geometry, then each photon contributes to casting a shadow (encoded flux) of part of the aperture

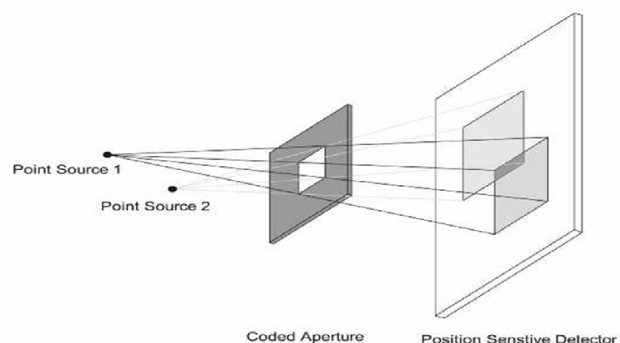

Figure 1: Schematic diagram of the CA, the production of a coded image and obtaining depth information. Increasing the source-aperture distance produces the smaller shadow of the CA on the detector. The lateral displacement of the shadow is proportional to that of the two sources (source plane). By correlating the recorded image with post processing, G function, an image of the source distribution at different depths may be determined. (a)

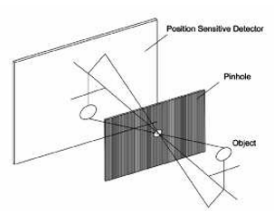

(b)

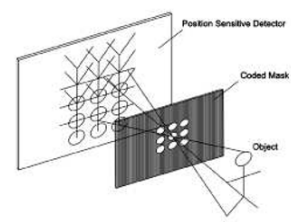

Figure 2: Schematic diagrams of two image formation principles: (a) a pinhole camera; the production of an image by a pinhole camera, (b) multiple pinholes camera (CA mask); the production of a coded image using signal multiplexing principle. Note: both techniques gives inverted image of the object.

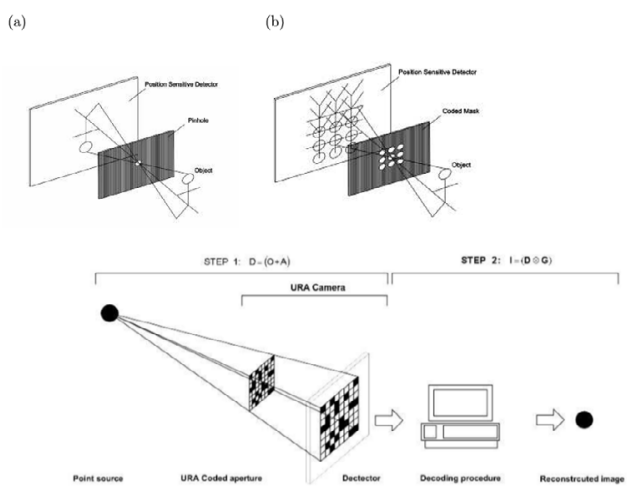

Figure 3: A schematic diagram of coded aperture principle imaging a point source.

pattern onto the detector surface. This means that the counts of the point source spread over a non-point like surface area. The size or magnification of the aperture shadow depends on the distance of the point source from the CA. To obtain a useful image, the encoded pattern is then decoded, most often by using the correlation of the observed pattern with a suitable decoding function.

In the case of imaging an extended object the basic concept does not change. Each point in the object can be regarded as a point source casting an aperture shadow of a certain size and location on the detector. In other words, the number of photons passing through a single hole of the CA is independent of photons passing through all other aperture holes. The contributions from each aperture hole represent the total projections of the object being imaged. These projections, collected at the detector, are composed of many shifted copies of the source object. The decoding process can be obtained in a similar way as in the case of point source. 


\section{Materials and Methods}

Other than experimental and MCS methods there are four possible methods for investigating the applications of CA for breast tumor imaging. This work introduces the initial and intrinsic method of investigation to represent the action of a distributed source in the projective CA imaging geometry. One of the main problems with MCS is that it takes very long execution time due to its intensive calculations. To obtain sufficient statistics the simulation can take several days and produce huge output files particularly with CA simulation. As an alternative two non-Monte Carlo methods, taking much less computation time, were developed to investigate and understand the performance of CA for imaging various source objects. The first method is referred to as the BMS whereas the second method is referred to as PRT. These methods are discussed below.

\section{Binary mask shift (BMS)}

This method was initiated using the projected image array of a perfect MUR binary mask generated by an on-axis point source. Then using assumed knowledge of the shape of the distributed source to be imaged, the projected pattern was systematically shifted according to the projected point source pattern that would be obtained by every non-zero element in the object. The result of each single or "binary shift" of the projected pattern is then summed with the preceding projection pattern. For illustration consider a binary mask that has been first shifted to the left then right and then up and down and finally summed.

$$
\left(\begin{array}{llll}
0 & 0 & 0 & 0 \\
0 & 1 & 1 & 0 \\
0 & 1 & 1 & 0 \\
0 & 0 & 0 & 0
\end{array}\right)\left(\begin{array}{llll}
0 & 0 & 0 & 0 \\
1 & 1 & 0 & 0 \\
1 & 1 & 0 & 0 \\
0 & 0 & 0 & 0
\end{array}\right)\left(\begin{array}{llll}
0 & 0 & 0 & 0 \\
0 & 0 & 1 & 1 \\
0 & 0 & 1 & 1 \\
0 & 0 & 0 & 0
\end{array}\right)\left(\begin{array}{llll}
0 & 1 & 1 & 0 \\
0 & 1 & 1 & 0 \\
0 & 0 & 0 & 0 \\
0 & 0 & 0 & 0
\end{array}\right)\left(\begin{array}{llll}
0 & 0 & 0 & 0 \\
0 & 0 & 0 & 0 \\
0 & 1 & 1 & 0 \\
0 & 1 & 1 & 0
\end{array}\right)\left(\begin{array}{llll}
0 & 1 & 1 & 0 \\
1 & 3 & 3 & 1 \\
1 & 3 & 3 & 1 \\
0 & 1 & 1 & 0
\end{array}\right)
$$

The summed shifted copies were then decoded with the usual postprocessing, G function (an inverse filter of A i.e. the mask). This binary mask displacement method represents a far-field approximation and is also given by using Equation 1 but with a constant $\left(\Omega\left(\vec{r}_{0}, \vec{r}_{m}\right)\right)$ term. This noise-less composite method was used to demonstrate the effect of artefacts from planar objects or displaced (shifted) sources. Mask transparency, finite mask thickness and statistical noise is not considered in this approach. But the projection data were convolved with a 2D Gaussian of standard deviation, $\sigma=1.57 \mathrm{~mm}$, to simulate the intrinsic PSF blurring of the gamma camera. As will be demonstrated in section 4, this simple BMS approach could be used to predict the source and the background pattern produced by uniform $2 \mathrm{D}$ object. This initial approach lead to the development of the Pseudo-Ray Tracing (PRT) method [30-32].

$$
\begin{aligned}
& D\left(\overrightarrow{r_{i}}\right)=\iint_{r_{0}} O\left(\vec{r}_{0}\right) A\left(\frac{a}{z} \vec{r}_{i}+\frac{b}{z} \vec{r}_{o}\right) \Omega\left(\overrightarrow{r_{o}}, \overrightarrow{r_{m}}\right) d^{2} \vec{r}_{o} \\
& \Omega\left(\overrightarrow{r_{o}}, \overrightarrow{r_{m}}\right)=\frac{p^{2} m}{a^{2}} \cos ^{3}(\theta)
\end{aligned}
$$$$
\text { where }: \theta=\arctan \left(\mid \vec{r}_{i}-\overrightarrow{r_{o} / z}\right) \text { and } p_{m} \text { is the mask pixel size }
$$

\section{Pseudo-ray tracing (PRT)}

This method used to investigate the background distortion observed when imaging planar and 3D source phantoms. The images obtained from the PRT are these generated from a phantom without a lesion. In all the projections of a CA-hole cast by a point on the object falling on the detector grid these are represented by Equation 1 and is calculated using purely geometric formulae. Simple in-house code was written to do this calculation based on calculating the fractional solid angle [33] subtended by each element of the mask whose shadow projected along the flux direction is intercepted by the detector. An accurate knowledge of the solid angle presented by the source $\Omega$ the mask is essential for calculating the projection. The solid angle, , (in steradian) is generally defined by an integral over the surface that faces the source. The calculation here is based on calculating the fractional solid angle subtended by a rectangle shape [33]. To illustrate this $\Omega$ :onsider the schematic diagram of Figure $2 \mathrm{a}$ the fractional solid angle, , subtended by the rectangle $0 \mathrm{BCD}$ at point $\mathrm{P}$ is represented by Equation 3:

$$
\Omega=h \int_{0}^{a} d x \int_{0}^{b} \frac{d y}{\left(x^{2}+y^{2}+h^{2}\right)^{\frac{3}{2}}}
$$

The above equation with the double integration can be solved and the result is as in Equation 4:

$$
\Omega=\arctan \frac{a b}{h \sqrt{\left(x^{2}+y^{2}+h^{2}\right)}}
$$

To illustrate how the fractional solid angles for each open element of the mask is calculated consider the schematic diagram of Figure $1 \mathrm{~b}$. The rectangle in this case is enclosed by the four straight lines $\mathrm{x}=\mathrm{x}_{1}$, $\mathrm{x}=\mathrm{x}_{2}, \mathrm{y}=\mathrm{y}_{1}$ and $\mathrm{y}=\mathrm{y}_{2}$ and the coordinates of the observation point $\mathrm{P}$ are $\left(x_{p}, y_{p}, z_{p}\right)$. For the small shaded area ABCD (see Figure $2 b$. The fractional solid angles, $\Omega$, is represented by four solid angles subtended by rectangles as in Equation 5:

$$
\begin{aligned}
& \Omega=\arctan \frac{\left(x_{2}-x_{p}\right)\left(y_{2}-y_{p}\right)}{z p\left[\left(x_{2}-x_{p}\right)^{2}+\left(y_{2}-y_{p}\right)^{2}+z_{p}\right]^{\frac{1}{2}}}-\arctan \frac{\left(x_{1}-x_{p}\right)\left(y_{2}-y_{p}\right)}{z p\left[\left(x_{2}-x_{p}\right)^{2}+\left(y_{2}-y_{p}\right)^{2}+z_{p}^{2}\right]^{\frac{1}{2}}} \\
& -\arctan \frac{\left(x_{2}-x_{p}\right)\left(y_{1}-y_{p}\right)}{z p\left[\left(x_{2}-x_{p}\right)^{2}+\left(y_{1}-y_{p}\right)^{2}+z_{p}\right]^{\frac{1}{2}}}+\arctan \frac{\left(x_{1}-x_{p}\right)\left(y_{1}-y_{p}\right)}{z p\left[\left(x_{1}-x_{p}\right)^{2}+\left(y_{1}-y_{p}\right)^{2}+z_{p}^{2}\right]^{\frac{1}{2}}}
\end{aligned}
$$

Equation 5 has been used to calculate the fractional solid angle subtended by each opening of the mask at an arbitrary point. To calculate the projection for a $2 \mathrm{D}$ or $3 \mathrm{D}$ object the solid angle calculations requires a priori knowledge of the source distribution. In SM this is not a major issue as the breast is commonly compressed to a known thickness, and the $2 \mathrm{D}$ projection can be obtained from a simple optical camera. The code finds the center of the projection of the mask-hole by ray-tracing (Figure 4 ).

\section{Results and Discussion}

In the last 15 years a number of attempts have been made to use (a)

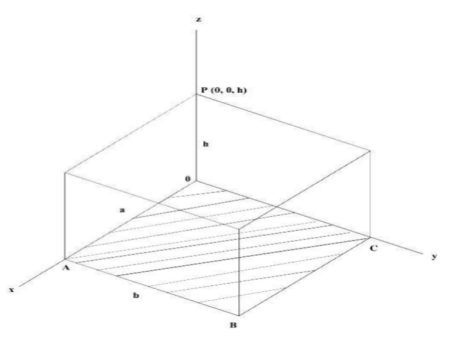

(b)

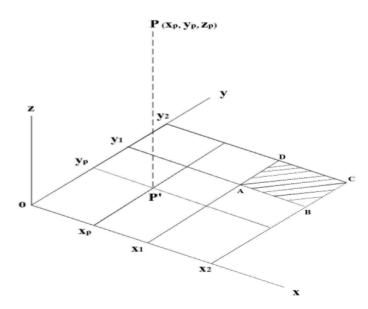

Figure 4: Schematic diagrams used to define the solid angle for a point $P$ : (a) a geometry for a solid angle subtended by the shaded rectangle OBCD, (b) a geometry for a solid angle subtended by the small shaded rectangle ABCD at the point $P$. Both geometrical diagrams were adapted from [33]. 
URA-CAs for 3D (SPECT and PET) imaging e.g. [34,35]. They have demonstrated that the use of URAs have improved sensitivity and resolution over single pinhole collimators. However, their resolution does not exceed that of planar parallel-hole collimators and this may be due to the complexity of the reconstruction or convolution algorithms for complex 3D imaging. To the best of the author's knowledge, no research other than $[30,36]$ has systematically investigated the application of CA for planar SM. This study thus, aims to continue the previous work by examining the state of CA imaging and systematically develops some of the theory of its application to breast tumour imaging. The major anticipated advantages and motivations. First, CA imaging is well suited for detecting faint pseudo-point like objects in nonzero background. Imaging point-like small lesions (objects) in medicine is akin to imaging stellar points (objects) in astronomy (Figure 5). Thus, CA imaging appears well matched to the imaging objectives in SM. Theoretically, the selected CA patterns (a)

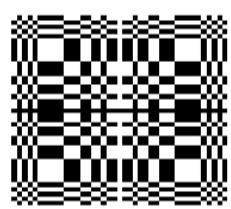

(c)

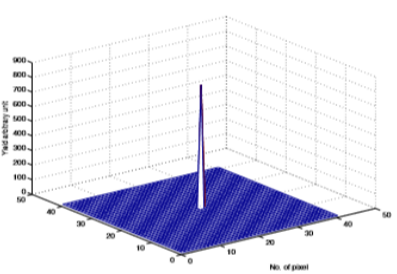

(b)

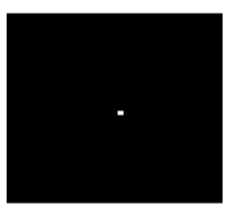

(a)

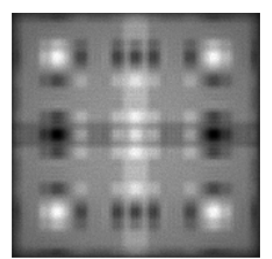

(c)

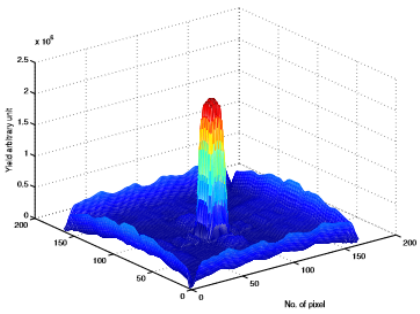

(b)

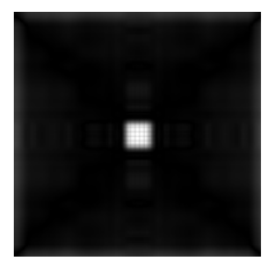

(d)

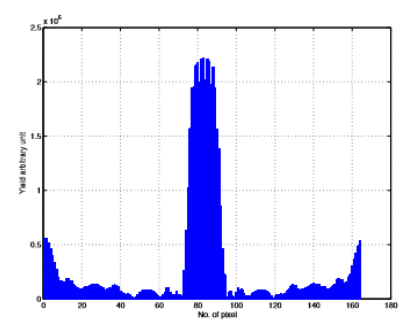

Figure 7: Exemplar plot of PRT of $1 \times 1 \mathrm{~cm}^{2}$ square source: (a) the projected image, (b) the decoded image, (c) a 3D plot of the decoded image, and (d) a vertical profile taken through the center of the decoded image. This should be compared with Fig. 6 . This technique successfully predicts the intrinsic flat field distortion caused by a distributed planar source.

have wide open area and thus about half of the incident $\gamma$-rays will be transmitted. This may dramatically improve the photon collection efficiencies and thus may reduce the acquisition time of the imaging system compared to a collimator-based system. Combining CAs using geometric magnification with a full size standard $\gamma$-camera for imaging a relatively small organ such as breast has a potential ability to maintain the resolution compared to using a conventional collimator. In addition, the hole size of the mask dominates the spatial resolution, making the CA-camera very attractive for early breast tumour imaging. Ultimately, high resolution could be achieved without the need for dedicated high resolution $\gamma$-camera instrumentations.

The two methods used specifically for CA imaging investigations. These are PRT method and a new but simple BMS method. These two iterative methods, namely PRT and BMS were developed for performing the CA imaging investigations and used as the basis for artefact prediction and correction. One of the key results from developing these approaches is that the form of the background artefact arises mainly due to the $2 \mathrm{D}$ distributed source geometry. For slightly compressed breasts $(6 \mathrm{~cm}$ compression), there are only minor contributions to the distributed activity artefact from depth activity. A study of the spatial variation in sensitivity was initiated using Binary Mask Shift (BMS) of the MURA pattern of $41 \times 41$ elements after being scaled by 4 demonstrated that the mask is basically shifted one pixel at a time to produce the required shifted point source. This noiseless composite mask was then decoded using the usual G function to demonstrate the intrinsic imaging properties of the MURA mask under consideration.

To consider the effects of all these geometrical artefacts, similar sized planar square objects were studied using the PRT method (Figure 6). The reconstructed image from such square source shown in Figure 7 was compared to the corresponding from the BMS (Figure 6). Both methods successfully predicted the flat field background distortion artefacts of the uniform 2D object imaged. With the PRT and MCS methods the radiation intensity reaching the detector are not uniform (solid angle effect) and this causes a sensitivity dip toward the center. Exemplar vertical profiles of predicted flat field distortion of $2 \mathrm{D}$ 
(a)

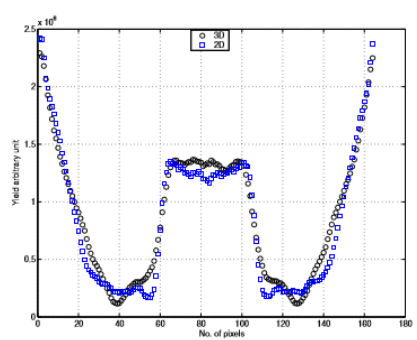

(b)

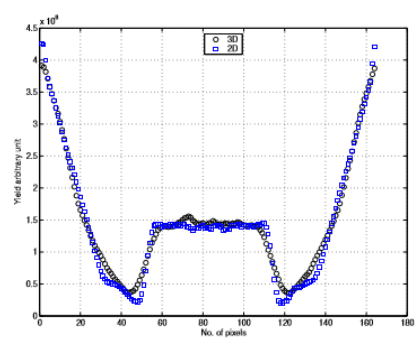

Figure 8: Exemplar vertical profiles of predicted 2D flat field distortion from the PRT method for a planar source compared with 3D source object after least squares fit to normalize the distribution: (a) planar square object of size $3 \times 3$ $\mathrm{cm}^{2}$ compared with 3D object of $3 \times 3 \times 6 \mathrm{~cm}^{3}$, (b) planar square object of size 4 $\times 4 \mathrm{~cm}^{2}$ compared with $3 \mathrm{D}$ object of $4 \times 4 \times 6 \mathrm{~cm}^{3}$.

square source object of size $1 \times 1 \mathrm{~cm}^{2}$ and $3 \times 3 \mathrm{~cm}^{2}$ from PRT method compared with the corresponding produced from MCS data is shown in Figure $8 \mathrm{a}$ and $8 \mathrm{~b}$ respectively. This show that the PRT method was found to produce flat field distortions that is almost identical to the MCS data reported elsewhere [36].

With the PRT method the radiation intensity reaching the detector is not uniform (solid angle effect) and this causes a sensitivity dip toward the center. Exemplar vertical profiles of predicted flat field distortion of 2D square source object of size $1 \times 1 \mathrm{~cm}^{2}$ and $3 \times 3 \mathrm{~cm}^{2}$ from PRT method. Now the PRT method is used to demonstrate the predicted flat field distortion obtained for different target volumes of fixed thickness $(6 \mathrm{~cm})$ ranging from $6-864 \mathrm{~cm}^{3}$. To consider the difference between a full 3D prediction of the background compare to just considering 2D in-plane effects, a high statistics PRT method was used. Figure 8 shows exemplar profiles of the decoded image of the predicted flat field distortion obtained from the PRT method for $2 \mathrm{D}$ planar and $3 \mathrm{D}$ volumetric source objects. The imaging profiles taken through the center of the decode images demonstrate that the flat field distortion artefacts from 3D volumetric source objects appear somehow similar to that observed when imaging a $2 \mathrm{D}$ planar object. It is also worth showing the decoded images resulting from these $2 \mathrm{D}$ planar and 3D volumetric source objects.

The 3D set of investigations suggests that the main cause of the distortion artefacts, in near-field geometry, is the finite distributed size of the source object mainly from within the focal plane. It also suggests that the form of the artefact, in terms of the shape and the magnitude of the side-lobes, arising from such imaging geometry can be predicted. These are encouraging results and suggest that the MURAs-CA near field distortion observed with distributed 3D source object, as might be found in SM, can be easily predicted and corrected.

\section{Conclusion}

The first method is attractive as it bases on a simple approach called BMS. Such approach allows the users to investigate all the possible built in artefacts without the effect of solid angle term. Thus, it provides understanding of the application of CA patterns in breast tumor imaging through the evaluation of their potential and their performance under a variety of imaging conditions. The main results demonstrate that MURAs CA patterns of squares arrays are very promising as it produces excellent decoded images. Thus, the technique is ideal and attractive for imaging small isolated sources and thus provides a good match to the imaging of SM. From the above investigations one may conclude that the MURA pattern for imaging a point-like object in the near field geometry has good performance with zero side lobes and

the image artefacts are intrinsic to CA imaging when using near-field geometry even when imaging small volumetric objects. In addition, the non-Monte Carlo methods i.e. the BMS and the PRT methods produce comparable results. Both methods successfully predict the form of artefacts, in term of the shape and the magnitude of the sidelobes that is due to the off-center source displacement. It should be noted that by even removing the effect of varying the incident $\gamma$-ray or solid angle effect (when using the BMS method), the artefacts are still present in the decoded image. These artefacts accounted for the effect of source displacement result in losing information by not covering the whole image. This suggests that for high resolution near-field imaging one ideally needs a large detector that fully samples the mask patterns for all the sources within the FoV. One of the observation demonstrated with MURA-CA investigations is that the out of-focus behavior of reconstruction has complex non-uniform structure. These investigations suggest that $3 \mathrm{D}$ imaging with $\mathrm{CA}$ is not the best option as the out of focus planes produces highly distorted images with severe artefacts. Distortion artefacts appear in the image with large magnitude once the geometry starts to become more complicated (distributed) and the FoV increases. These were visualized for objects larger than 1 $\times 1 \mathrm{~cm}^{2}$. The source of background distortion artefacts appears when imaging 2D and 3D object are believed to be due to a combination of several factors: 1) Due to finite size of the detector the off-axis sources cause incomplete (partial coding) of the mask pattern. 2) The inherent non-linearity in the photon flux impinging on the detector. The nonMonte Carlo methods successfully predict the form of artefacts arising from imaging extended objects of $2 \mathrm{D}$ shape. The BMS method predicted the shape of artefacts that are due to off-axis sources and finite size of the object (extended sources) but ignores the effect of varying the angle of incidence of the gamma rays. However, for PRT method the near field effect cause non-uniform radiation intensity to reach the detector and thus another form of artefacts appear in the image. The background artefacts produced by uniform $2 \mathrm{D}$ and $3 \mathrm{D}$ source objects of different sizes using the PRT method encouraging and thus, the so-called near field distortion observed with distributed planar and 3D sources, as might be found in SM using coded apertures, can be easily predicted and corrected [32]. The predicted background can be used to correct the near-field effect of 3D sources, as might be found in SM using CA. To apply the background subtraction correction, the shape and the thickness of the breast needs to be identified. In practice the thickness of this is known from the amount of compression used, typically $6 \mathrm{~cm}$. The shape could then be easily captured from an optical image acquired from above compressed breast. Once this information is available then MCS or the PRT method, which is faster, can be used to produce a prediction of the expected background distributed radioisotope distribution.

\section{References}

1. Siegel R, Naishadham D, Jemal A (2013) Cancer statistics, 2013. CA Cancer J Clin 63: 11-30.

2. DeSantis C, Ma J, Bryan L, Jemal A (2014) Breast Cancer statistics, 2013. CA Cancer J Clin 64: 52-62.

3. Taillefer R (1999) The role of 99m Tc-sestamibi and other conventional radiopharmaceuticals in breast cancer diagnosis. Semin Nucl Med 29: 16-40.

4. Medical Advisory Secretariate Ontario ministry of health and long-term care," Scintimammography" (2003) Toronto: Ontario ministry of health and long-term care.

5. Tolmos J, Cutrone JA, Wang B, Vargas HI, Stuntz M (1998) Scintimammography analysis of non-palpable breast lesions previously identified by conventional mammography. JNCI 90: 846-849.

6. Derebek E, Balci P, Alanyali H, Kocdor MA, Capa G, et al. (1999) Detection of bilateral multifocal breast cancer using ${ }^{99 \mathrm{~m} T c-s e s t a m i b i}$ imaging: The role of delayed imaging. Clin Nucl Med 24: 590-593. 
Citation: Alnafea MA, Mahboub D, Wells K (2017) Non-Monte Carlo Methods for Investigating the Application of Coded Aperture Breast Tumour Imaging. J Cancer Sci Ther 9: 748-754. doi:10.4172/1948-5956.1000503

7. Taillefer R (2005) Clinical application of ${ }^{99 m} \mathrm{Tc}$-sestamibi scintimammography. Seminars in Nuclear Medicine 35: 100-115.

8. Spanu A, Dettori G, Nuvoli S, Porcu A, Falchi A, et al. (2001) 99mTc-tetrofosmin SPET in the detection of both primary breast cancer and axillary lymph node metastasis. Eur J Nucl Med 28: 1781-1794.

9. Itti E, Ahdoot $\mathrm{H}$, Khalkhali I (2002) Scintimammography for the diagnosis of breast cancer. J Wom Imag 4: 66-72.

10. Bruening W, Launders J, Pinkney N, Kostinsky H, Schoelles K (2006) Effectiveness of non-invasive diagnostic tests for breast abnormalities: Comparative effectiveness review.

11. Scopinaro F, Pani R, De Vincentis G, Soluri A, Pellegrini R (1999) High resolution scintimammography improves the accuracy of technetium-99m methoxy isobutylisonitrile scintimammography: Use of a new dedicated gamma camera. Eur J Nucl Med 40: 1279-1288.

12. Majewski S, Kieper D, Keppel C (2000) Optimization of dedicated scintimammography procedure using small detector prototypes and compressible breast phantoms. Presented at the IEEE Medical Imaging Conference, Lyon, France.

13. Coover LR, Caravaglia G, Kunh P (2004) Scintimammography with dedicated breast camera detects and localizes occult carcinoma. J Nucl Med 45: 553-558.

14. Brem RF, Schoonjans JM, Kieper DA, Majewski S, Goodman S (2002) Highresolution scintimammography: a pilot study. J Nucl Med 43: 909-915.

15. Brem RF, Schoonjans JM, Kieper DA, Majewski S, Goodman S (2003) Evaluation of a high resolution, breast-specific, small-field-of-view gamma camera for the detection of breast cancer. Nucl Instrum Methods Phys Res A 497: 39-45.

16. Rhodes DJ, O'Connor MK, Phillips SW, Smith RL, Collins DA (2005) Molecular breast imaging: A new technique using ${ }^{99 \mathrm{~m} T c}$ - scintimammography to detect small tumours of the breast. Mayo Clinic Proceedings 80: 24-30.

17. Brem RF, Rapelyea JA, Zisman G, Mohtashemi K, Raub J (2005) Occult breas cancer: Scintimammography with high-resolution breast-specific gamma camera in women at high risk for breast cancer. Radiology 237: 274-280.

18. Barrett HH (1972) Fresnel Zone Plate Imaging in Nuclear Medicine. J Nucl Med 4: 382-385.

19. Dicke RH (1968) Scatter-hole Cameras for X-rays and gamma Ray. Astrophysical J 153: L101-L106.

20. Webb S (1998) The Physics of Medical Imaging, Institute of Physics Publishing, Bristol and Philadelphia, USA

21. Barrett HH, Swindell W (1981) Radiological imaging: the theory of image formation, detection and Processing, Academic Press London, UK.
22. Cannon TM, Fenimore EE (1979) Tomographic imaging using uniformly redundant arrays. Applied Optics 18: 1052-1057.

23. Fenimore EE, Cannon TM (1997) Uniformly redundant arrays. Proceeding Digital Signal Processing Symposium 6: 479-493.

24. Fenimore EE, Cannon TM (1978) Coded aperture imaging with uniformly redundant array. Applied Optics 17: 337-347.

25. Gottesman SR, Fenimore EE (1989) New family of binary arrays for coded aperture imaging. Applied Optics 28: 4344-4352.

26. Fenimore EE, Cannon TM (1981) Uniformly redundant arrays: digital reconstruction methods. Applied Optics 20: 1858-1864.

27. Fenimore EE (1978) Coded aperture imaging: predicted performance of uniformly redundant array. Applied Optics 17: 3562-3570.

28. Meikle SR, Fulton RR, Eberl S, Dahlbom M, Wong K (2001) An investigation of coded aperture imaging for small animal SPECT. IEEE Transactions on nuclear science 3: 816-821.

29. Dunphy P, McConnell M, Owens A, Chupp E, Forrest D, et al. (1989) A balloonborne coded aperture telescope for low-energy gamma-ray astronomy. Nucl Instrum Methods Phys Res A 274: 362-379.

30. Alnafea M, Wells K, Spyrou NM, Saripan MI Guy M (2006) Preliminary results from a Monte Carlo study of breast tumour imaging with low energy highresolution collimator and a modified uniformly-redundant array coded aperture. Nucl. Instrum. Methods Phys Res Sect A Accel Spectrom Detect Asspc Equip 63: 146-149.

31. Alnafea MA, Wells K, Spyrou NM, Guy M (2007) Preliminary Monte Carlo study of coded aperture imaging with a CZT gamma camera system for scintimammography. Nucl Instrum Methods Phys Res A 573: 122-125.

32. Alnafea MA, Wells K, Spyrou NM, Guy M (2007) Near field correction for coded aperture Imaging in scintimammography" IEEE Xplore.

33. Gotoh H, Yangi H (1971) Solid angle subtended by a rectangular slit. Nuclear Instrument and Method in physics reasach 221: 56-59.

34. Gemmill PE, Chaney RC, Fenyves EJ (1995) Monte-Carlo simulation of a coded aperture SPECT apparatus using uniformly redundant arrays. Imaging detectors in high energy and astroparticle physics, Los Angeles, CA, Singapore 81-115.

35. Zhang L, Lanza RC, Horn BKP, Zimmerman RE (1998) Three-dimensional coded aperture techniques in diagnostic nuclear medicine imaging. SPIE Conference on Physics of Medical Imaging 3336: 364-373.

36. Michal J, Cieslak, Kelum AA, Gamage, Robert G (2016) Coded aperture imaging system: Past, present and future. Radiation Measurements 92: 59-71. 